\title{
LOCAL COHOMOLOGY MODULES AND RELATIVE COHEN-MACAULAYNESS
}

\author{
M. Mast Zohouri \\ Department of Mathematics \\ Payame Noor University \\ Tehran, 19395-3697, Iran
}

e-mail: m.mast.zohouri@gmail.com

\begin{abstract}
Let $(R, \mathfrak{m})$ denote a commutative Noetherian local ring and let $M$ be a finite $R$-module. In this paper, we study relative Cohen-Macaulay rings with respect to a proper ideal $\mathfrak{a}$ of $R$ and give some results on such rings in relation with Artinianness, Non-Artinianness of local cohomology modules and Lyubeznik numbers. We also present some related examples to this issue.
\end{abstract}

Keywords: local cohomology modules, Lyubeznik numbers, Non-Artinian modules, relative Cohen-Macaulayness.

2010 Mathematics Subject Classification: Primary 13D45; Secondary 13C14, 13E10.

\section{REFERENCES}

[1] M.P. Brodmann and R.Y. Sharp, Local Cohomology: an Algebraic Introduction with Geometric Applications (Cambridge University Press, Cambridge, 2013).

[2] CoCoATeam, CoCoA: A system for doing computation in Commutative Algebra, cocoa.dima.unige.it.

[3] M.T. Dibaei and A. Vahidi, Artinian and non-Artinian local cohomology modules, Canad. Math. Bull. 54 (2011) 619-629. doi:10.4153/CMB-2011-042-5

[4] M.T. Dibaei and S. Yassemi, Associated primes and cofiniteness of local cohomology modules, Manuscripta Math. 117 (2005) 199-205. doi:10.1007/s00229-005-0538-5

[5] M. Eghbali, On set theoretically and cohomologically complete intersection ideals, Canad. Math. Bull. 57 (2014) 477-484. 
[6] V. Erdogdu and T. Yildirim, On the cohomological dimension of local cohomology modules, arXiv:1504.01148v2 [math.AC].

[7] R. Hartshorne, Affine duality and cofiniteness, Invent. Math. 9 (1970) 145-164. doi:10.1007/BF01404554

[8] J. Herzog, Komplex Auflosungen and Dualitat in der Lockalen Algebra, Habilitationss chrift (Universitat Regensburg, 1970).

[9] L. Melkersson, Some applications of a criterion for artinianness of a module, J. Pure Appl. Algebra 101 (1995) 291-303. doi:10.1016/0022-4049(94)00059-R

[10] M. Mast Zohouri, KH. Ahmadi Amoli, and S.O. Faramarzi, Relative CohenMacaulay filtered modules with a view toward relative Cohen-Macaulay modules, Math. Reports 20 (70), 3 (2018).

[11] G. Lyubeznik, Finiteness properties of local cohomological modules (an application of D-modules to Commutative Algebra), Invent. Math. 113 (1993) 41-55. doi:10.1007/BF01244301

[12] R. L $\ddot{u}$ and Z. Tang, The f-depth of an ideal on a module, Proc. Amer. Math. Soc. 130 (2001) 1905-1912. doi:10.1090/S0002-9939-01-06269-4

[13] A. Ooishi, Matlis duality and the width of a module, Hiroshima Math. J. 6 (1976) 573-587. projecteuclid.org/euclid.hmj/1206136213.

[14] M. Rahro Zargar, Some duality and equivalence results, arXiv:1308.3071v2 [math.AC].

[15] A.S. Richardson, Co-localization, co-support and local cohomology, Rocky Mountain J. Math. 36 (2006) 1679-1703. doi:10.1216/rmjm/1181069391

[16] N. Suzuki, On the generalized local cohomology and its duality, J. Math. Kyoto Univ. 18 (1978) 71-85.

doi:10.1215/kjm/1250522630

Received 21 March 2018

Revised 5 July 2018

Accepted 12 July 2018 